\title{
Evidence for Hydroxyl Radical Involvement in Group B Streptococcus-Induced Pulmonary Hypertension and Arterial Hypoxemia in Young Piglets
}

\author{
THOMAS H. PAULY, BRUCE D. BOWDY, CHERYL A. HAVEN, STEPHANIE B. BARR, AND \\ MARK N. GILLESPIE \\ University of Kentucky A.B. Chandler Medical Center, College of Medicine, Department of Pediatrics, Division of \\ Neonatology [T.H.P., S.B.B.] and College of Pharmacy, Division of Pharmacology and Toxicology [B.D.B., \\ C.A.H., M.N.G.], Lexington, Kentucky 40536
}

\begin{abstract}
Early onset neonatal GBS infection is associated with pulmonary hypertension, pulmonary edema, and arterial hypoxemia. Although the mechanisms underlying these cardiopulmonary disturbances are not completely understood, multiple lines of evidence suggest that inflammatory mediators may be involved. This study examined the actions of dimethylthiourea (DMTU), a relatively selective scavenger of hydroxyl radical, on GBSinduced pulmonary hypertension, arterial hypoxemia, and pulmonary edema formation in young piglets. Relative to control animals, intravenous infusion of GBS $\left(10^{8}\right.$ organisms $/ \mathrm{kg} / \mathrm{min}$ for $60 \mathrm{~min}$ ) provoked sustained increases in pulmonary arterial pressure (Ppa: $\mathbf{+ 8 8 \%}$ ) and total pulmonary resistance (TPR: $128 \%$ ). GBS infusion also was associated with profound decreases in arterial $\mathrm{PO}_{2}(-58 \%)$. Pulmonary edema was present in GBS-treated animals as evidenced by an $8.4 \%$ increase in the lung wet-to-dry weight ratio. After pretreatment with DMTU $(0.75 \mathrm{~g} / \mathrm{kg}$ administered intravenously over $30 \mathrm{~min}$ ), GBS increased Ppa by $33 \%$ and TPR by only $16 \%$. Similarly, after DMTU pretreatment GBS decreased arterial oxygen tension by only $12 \%$. DMTU also limited the GBS-induced increase in lung wet-to-dry weight ratio to $2.6 \%$ These findings demonstrate that DMTU attenuates GBS-induced pulmonary hypertension, pulmonary edema, and arterial hypoxemia and suggest that hydroxyl radicals play an important role in these cardiopulmonary disturbances. (Pediatr Res 24: 735-739, 1988)
\end{abstract}

\section{Abbreviations}

CI, cardiac index

DMTU, dimethylthiourea

GBS, group B streptococci

Ppa, mean pulmonary arterial pressure

Psa, mean systemic arterial pressure

TPR, total pulmonary resistance

TSR, total systemic resistance

A major consequence of early onset neonatal GBS infection is profound pulmonary hypertension and arterial hypoxemia. Al-

Received March 21, 1988; accepted August 12, 1988.

Correspondence Mark N. Gillespie, Ph.D., University of Kentucky, College of Pharmacy, Division of Pharmacology and Toxicology, Lexington, KY 40536-0082.

Supported in part by grants from the National Institutes of Health (HL-38495, HL-36404, HL-02174), the Cystic Fibrosis Foundation, and the American Heart Association, Kentucky Affiliate. though the mechanism(s) underlying the adverse cardiopulmonary effects of GBS infection are not completely understood, recent experiments in animal models have provided compelling support for involvement of eicosanoids. For example, in GBStreated piglets, both cyclooxygenase and thromboxane $A_{2}$ synthetase inhibition (1-3) forestall development of cardiopulmonary abnormalities. Blockade of peptidoleukotriene receptors also has been shown to exert salutary effects on GBS-induced hemodynamic and bronchomotor responses (4-6). Along with these mediators, numerous studies in adult animals have suggested that toxic oxygen radicals play a central role in development of acute lung injury (7). Involvement of oxygen radicals in GBS-induced cardiopulmonary disturbances has not been examined.

The present studies evaluated the contribution of hydroxyl radicals to development of GBS-induced lung injury and pulmonary hypertension in young piglets. We reasoned that if hydroxyl radicals played a central role, then a scavenger of this oxidant species, DMTU, should attenuate the adverse cardiopulmonary consequences of GBS. We focused our efforts on hydroxyl radicals and on DMTU for several interrelated reasons. First, hydroxyl radicals seem to be the most toxic of the oxygen radical species (7) and are believed to play causative roles in the acute lung injury provoked in adult animal models by endotoxin, phorbol myristate acetate, hyperoxia, and thiourea (8-10). Second, because DMTU is a reasonably selective scavenger of hydroxyl radicals (10), beneficial effects of this agent are strongly suggestive of hydroxyl radical involvement. Third, inasmuch as DMTU is a small, relatively lipophilic molecule, it has the potential to cross cell membranes and thereby scavenge intracellularly generated hydroxyl radicals. This latter feature may be important if intracellular generation of oxidants is a key component of lung injury in the setting of GBS-induced sepsis as it may be in other models of lung injury $(11,12)$.

\section{MATERIALS AND METHODS}

Animal model. Twenty-four young piglets unselected as to gender and ranging in age and weight from 6 to 14 days and 2 to $4.5 \mathrm{~kg}$, respectively, were randomly assigned to four experimental groups as described below. Animals were anesthetized with an intraperitoneal injection of $30 \mathrm{mg} / \mathrm{kg}$ sodium pentobarbital and placed on a heat-exchanging pad to maintain body temperature at $38^{\circ} \mathrm{C} \pm 1^{\circ}$. Catheters were introduced to permit the following determinations and interventions: Psa was measured from a catheter inserted into the left femoral artery; Ppa was measured from a specially formed polyethylene catheter inserted into the right external jugular vein and advanced into the pulmonary artery; for determination of $\mathrm{CI}$ by thermodilution 
(Edwards Laboratory model 9520A thermodilution cardiac output computer, Santa Anna, CA), $1.0 \mathrm{ml}$ ice-cold saline was injected into a catheter introduced into the right femoral vein and advanced to the level of the right atrium while changes in blood temperature were detected by a $5 \mathrm{~F}$ thermistor-tipped, double lumen catheter inserted into the left carotid artery and advanced to the aortic arch; arterial blood samples for blood gas determination were taken from a lumen of the thermistor-tipped catheter; DMTU or its vehicle (isotonic saline) was infused into the left femoral vein; and GBS or its vehicle was infused into the right external jugular vein.

Psa and Ppa were monitored using Statham P23 pressure transducers (Gould, Inc., Oxnard, CA) zeroed at mid-thoracic level in conjunction with a Grass model 7 polygraph (Grass Instruments, Quincy, MA). Arterial blood gases and $\mathrm{pH}$ were determined in $0.8 \mathrm{ml}$ blood samples using an Instrument Laboratories model 213 blood-gas analyzer (Lexington, MA). The following computations were performed: TPR was calculated as the quotient of Ppa and CI; TSR was calculated as the quotient of Psa and CI. In cases where multiple cardiac index measurements were obtained per time point, the averaged value was used in the above calculations.

After catheter introduction, a tracheostomy was performed, and the animals were connected to a Harvard small animal ventilator. The animals were then paralyzed with pancuronium bromide $(0.75 \mathrm{mg} / \mathrm{kg}$ intravenous followed by supplemental doses of $0.4 \mathrm{mg} / \mathrm{kg}$ as needed) and ventilated at $50 \mathrm{breaths} / \mathrm{min}$ with a mixture of $30 \% \mathrm{O}_{2}$ in $\mathrm{N}_{2}$, using a tidal volume of $7 \mathrm{ml} /$ $\mathrm{kg}$. At the onset of a 20-min acclimatization period, ventilator settings were adjusted to attain baseline arterial $\mathrm{PO}_{2}, \mathrm{PCO}_{2}$, and $\mathrm{pH}$ of approximately 100 torr, 40 torr, and 7.4, respectively. Ventilator settings were not changed during the subsequent experimental protocols.

Upon termination of each experiment, the animals were killed with a lethal dose of i.v. sodium pentobarbital, and the lungs were excised rapidly. After the right lung was blotted on filter paper to remove adherent blood and extrapulmonary tissue was dissected free, it was weighed wet and then dried at $70^{\circ} \mathrm{C}$ until a stable dry weight was obtained. As validated by Collins et al. (13), the ratio of blood inclusive wet weight to the blood inclusive dry weight was taken as an index of pulmonary edema formation.

Bacterial preparation. Cultures of GBS (Streptococcus agalacticae: American-type Culture Collection, Difco Laboratories, Detroit, MI) were grown to late log phase in 500-ml aliquots of Todd-Hewitt broth (Difco Laboratories). The broth was then centrifuged at $2500 \times g$ for $30 \mathrm{~min}$ and the resulting pellet resuspended in D5 Ringer's lactate solution to a final bacterial concentration of approximately $1 \times 10^{9}$ organisms $/ \mathrm{ml}$. Bacterial concentration was determined using conventional quantitative culture techniques by relating colony-forming units to optical density. Bacteria were stored at $4^{\circ} \mathrm{C}$ and used within $1 \mathrm{wk}$ of preparation. Viability at the time of use was routinely more than $70 \%$.

Experimental protocol. Four groups of six animals were studied. One group served as control and received a 60-min infusion of the GBS vehicle, D5 Ringer's lactate solution, plus a 30-min infusion of sterile saline, the vehicle for DMTU. The total volume of infusates was 18 to $20 \mathrm{cc}$, depending on the weight of the animal. The DMTU-vehicle infusion was initiated $15 \mathrm{~min}$ prior to GBS-vehicle administration. A second group of animals received a 60 -min infusion of approximately $1 \times 10^{8} \mathrm{GBS} / \mathrm{kg} / \mathrm{min}$ plus the vehicle for DMTU, administered as described above. The third group received the vehicle for GBS plus $0.75 \mathrm{~g} \mathrm{DMTU/}$ $\mathrm{kg}$ over a 30 -min infusion period. DMTU was purchased from (Aldrich Chemicals Co. Milwaukee, WI). Finally, the fourth group received GBS plus DMTU. Hemodynamic and blood gas parameters were determined at 10 -min intervals beginning 20 min prior to GBS and continuing for the 60 -min infusion period.

Statistical analysis. Time and experimental group-dependent differences in hemodynamic and blood gas parameters were evaluated using a 2-way analysis of variance combined with Newman-Keuls' test for multiple comparisons when appropriate. Experimental group-dependent differences in the lung wet-todry weight ratio were evaluated using a one-way analysis of variance combined with Newman-Keuls' test for multiple comparisons. In both cases, $p$ values $\leq 0.05$ were considered to denote statistical significance. Data are presented as the mean $\pm \mathrm{SE}$.

\section{RESULTS}

Baseline hemodynamic parameters. Baseline hemodynamic parameters are shown in Table 1 . There were no differences among the four experimental groups in terms of baseline mean pulmonary or systemic arterial pressures. However, baseline CI was significantly greater in animals assigned to the GBS and GBS plus DMTU treatment groups than in control animals or animals receiving DMTU. These differences in CI were translated into significantly decreased baseline TPR in animals to be assigned to the GBS treatment group. Because of these differences in baseline values, data regarding the effects of GBS and DMTU, given alone or in combination, are depicted graphically in terms of time-dependent changes from baseline. However, results of statistical analyses performed on data transformed in this manner and on absolute data are provided below.

Effects of DMTU on GBS-induced changes in hemodynamic parameters. The effects of GBS and DMTU, alone and in combination, on changes in Ppa, Psa, and CI are shown in Figure 1. Although Ppa was stable in control animals and animals treated with DMTU, animals receiving GBS exhibited a significant 30-torr increase which was sustained over the $60-\mathrm{min}$ observation period. This increase was significantly attenuated by DMTU; Ppa in animals treated concomitantly with GBS plus the hydroxyl radical scavenger increased initially by 18 torr and then declined by 20 min to a level that was 5 to 10 torr above the preinfusion baseline. Changes in Psa exhibited by the four experimental groups did not attain statistical significance, although there was a trend for an increase in Psa in GBS-treated piglets that was not observed in animals receiving GBS plus DMTU. Statistical analyses applied to absolute pulmonary and systemic arterial pressures in the four experimental groups revealed effects of DMTU identical to those described above: DMTU significantly attenuated the rise in Ppa provoked by GBS. Similarly, there were no significant inter-group differences in the response of Psa to GBS or DMTU. Also, as shown in Figure 1, GBS-treated animals exhibited significant decreases in CI which were attenuated by concomitant administration of DMTU at all times except the 30 -min point. However, when absolute data were analyzed, differences between experimental groups did not attain statistical significance, although the same trends for a GBS-induced reduction in $\mathrm{Cl}$ and protection by concomitant treatment with DMTU were evident.

The actions of GBS and DMTU, alone and in combination, on changes in calculated TPR and TSR are shown in Figure 2. Group B streptococci caused a significant increase in TPR which was apparent as early as 10 min after initiation of infusion and

Table 1. Baseline hemodynamic parameters in young piglets assigned to control group or groups to be treated with DMTU, $G B S$, or GBS plus DMTU

\begin{tabular}{cccccc}
\hline $\begin{array}{c}\text { Experimental } \\
\text { group }\end{array}$ & $\begin{array}{c}\text { Ppa } \\
(\mathrm{mm} \mathrm{Hg})\end{array}$ & $\begin{array}{c}\text { Psa } \\
(\mathrm{mm} \mathrm{Hg})\end{array}$ & $\begin{array}{c}\mathrm{CI} \\
(\mathrm{ml} / \mathrm{min} / \mathrm{kg})\end{array}$ & $\begin{array}{c}\text { TPR } \\
(\mathrm{U} / \mathrm{kg})\end{array}$ & $\begin{array}{c}\text { TSR } \\
(\mathrm{U} / \mathrm{kg})\end{array}$ \\
\hline Control & 21.3 & 81.6 & 121.6 & 0.177 & 0.688 \\
& \pm 1.3 & \pm 6.2 & \pm 7.7 & \pm .011 & \pm .072 \\
DMTU & 17.4 & 77.7 & 138.3 & 0.128 & 0.573 \\
& \pm 0.8 & \pm 3.9 & \pm 6.8 & \pm .009 & \pm .050 \\
GBS & 16.6 & 78.8 & $163.0^{*}$ & $0.106^{*}$ & 0.493 \\
& \pm 1.5 & \pm 2.9 & \pm 8.6 & \pm .014 & \pm .034 \\
GBS + & 21.2 & 81.8 & $160.8^{*}$ & 0.138 & 0.524 \\
DMTU & \pm 2.8 & \pm 6.4 & \pm 6.4 & \pm .021 & \pm .051 \\
\hline
\end{tabular}

* Different from control and DMTU groups at $p<0.05$. 

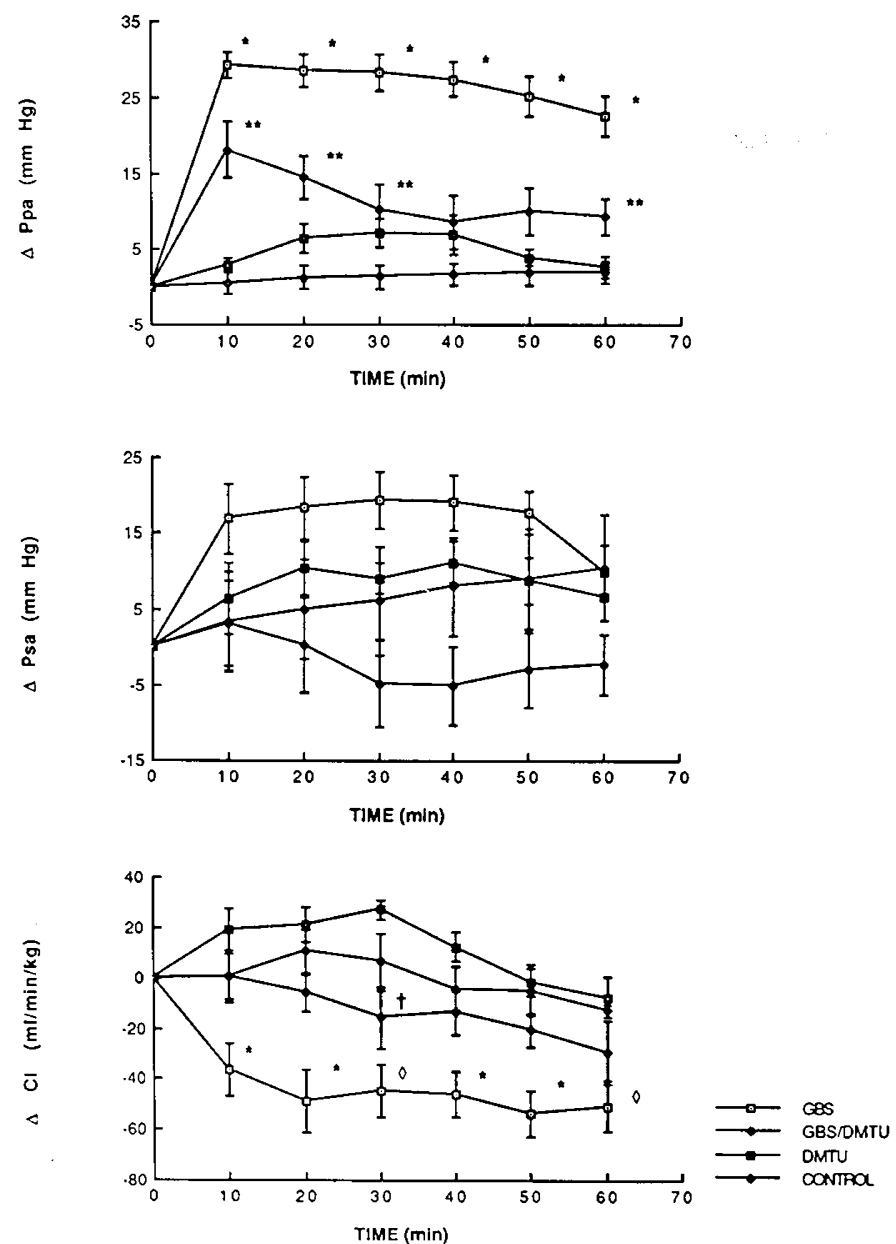

Fig. 1. Changes in mean pulmonary arterial pressure (Ppa: top panel), mean systemic arterial pressure (Psa: middle panel) and cardiac index (CI: bottom panel) expressed as a function of time in control animals and in animals treated with GBS, DMTU, or GBS plus DMTU. $n=6$ for each group. Data are expressed as the mean \pm SEM. ${ }^{*}$ Different from all other groups at $p<0.05 ; * *$ different from both control and GBS at $p<0.05 ;{ }^{\diamond}$ different from both control and DMTU at $p<0.05 ; \uparrow$ different from DMTU at $p<0.05$.

sustained for the duration of the observation period. The increase was reduced by approximately $66 \%$ when GBS and DMTU were co-administered. Analysis of absolute TPR, as opposed to changes in TPR from baseline, revealed identical results: DMTU attenuated the increase normally provoked by GBS. Changes in TSR followed a similar pattern. GBS-treated animals exhibited sustained increases which were abolished by concomitant administration of DMTU. Analysis of absolute TSR data indicated that the increases at 10 and $20 \mathrm{~min}$ postGBS were attenuated but not abolished by DMTU, whereas differences between GBS and GBS plus DMTU-treated animals occurring after $30 \mathrm{~min}$ of infusion did not attain statistical significance.

Effects of DMTU on GBS-induced changes in arterial blood gases and lung water. The effects of DMTU on GBS-induced changes in arterial $\mathrm{PO}_{2}, \mathrm{PCO}_{2}$, and $\mathrm{pH}$ are shown in Figure 3. Piglets infused with GBS exhibited profound decreases in $\mathrm{PO}_{2}$ which were sustained throughout the 60 -min observation period. Dimethylthiourea abolished these changes: arterial $\mathrm{PO}_{2}$ did not differ significantly between control animals, animals treated with DMTU, and animals treated with GBS plus DMTU. Although time-dependent differences in arterial $\mathrm{PCO}_{2}$ among the four experimental groups did not attain statistical significance, animals receiving GBS tended to become hypercarbic. Similar trends were not observed for the other experimental groups, notably
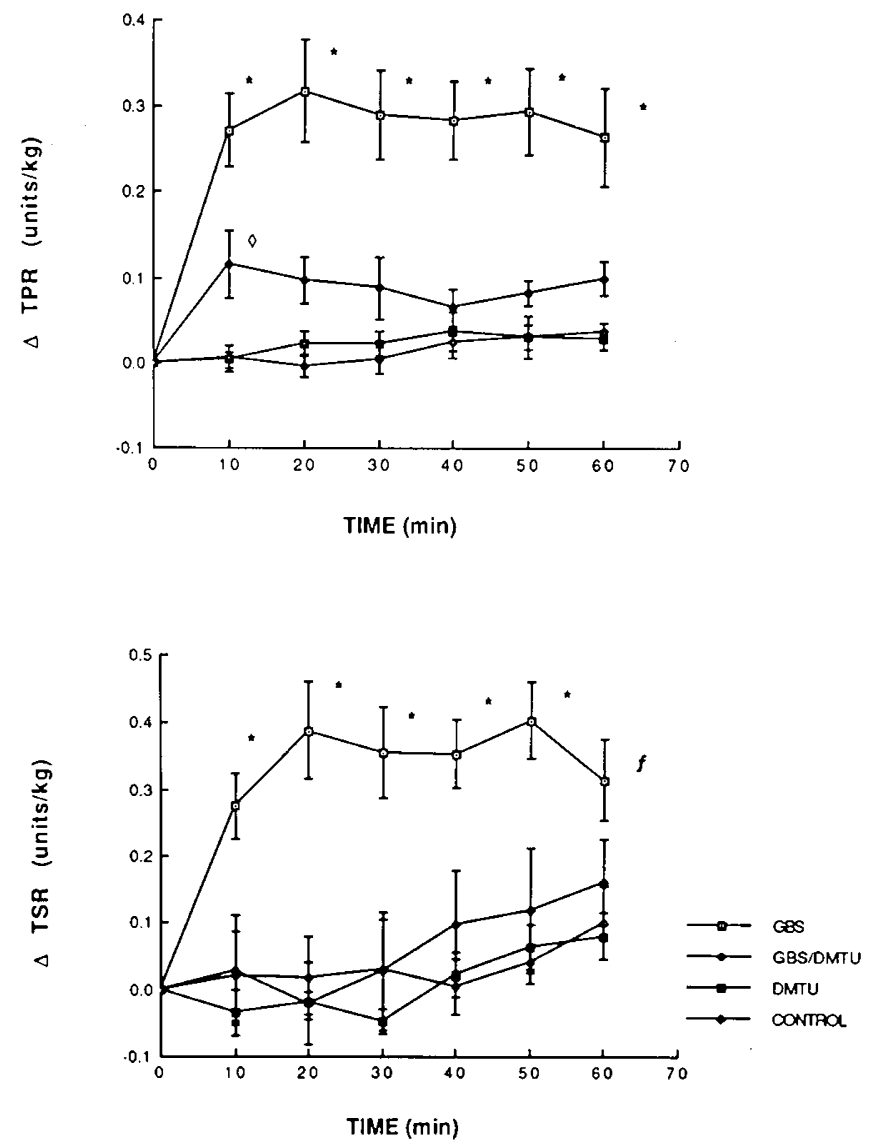

Fig. 2. Changes in total pulmonary resistance (TPR: top panel) and total systemic resistance (TSR: bottom panel) expressed as a function of time in control animals and in animals treated with GBS, DMTU, or GBS plus DMTU. $n=6$ for each group. Data are expressed as the mean \pm SEM. ${ }^{*}$ Different from all other groups at $p<0.05 ;^{\circ}$ different from both control and DMTU at $p<0.05 ;{ }^{f}$ different from both GBS and GBS/DMTU-treated animals at $p<0.05$.

the animals receiving GBS and DMTU concomitantly. Arterial $\mathrm{pH}$ decreased significantly within $20 \mathrm{~min}$ in GBS-treated animals. Neither control animals, animals treated with DMTU alone, nor animals receiving GBS plus DMTU exhibited timedependent changes in arterial $\mathrm{pH}$.

As illustrated in Figure 4, group B streptococcus infusion was associated with modest accumulation of lung water as evidenced by an $8.4 \%$ increase in lung wet-to-dry weight ratio. Although DMTU itself failed to influence the ratio, the increase normally caused by GBS infusion was limited to $2.6 \%$ in animals treated simultaneously with GBS plus DMTU.

\section{DISCUSSION}

The prominent observations of this study are that treatment of young piglets with DMTU attenuates development of GBSinduced pulmonary hypertension, arterial hypoxemia, and pulmonary edema. Although spurious inconsistencies in baseline CI values complicated data interpretation to some extent, the protective actions of DMTU were apparent regardless of whether the statistical analyses focused on changes from baseline or on absolute values. Inasmuch as DMTU is a relatively selective scavenger of hydroxyl radicals (10), our findings support the conclusion that this oxidant species plays a central role in the cardiopulmonary consequences of GBS septicemia. The young piglet with GBS-induced pulmonary hypertension and lung injury thus joins a rather extensive list of experimental models of acute respiratory distress in which hydroxyl radicals can be implicated on the basis of the salutary effects of DMTU $(8-10)$. 

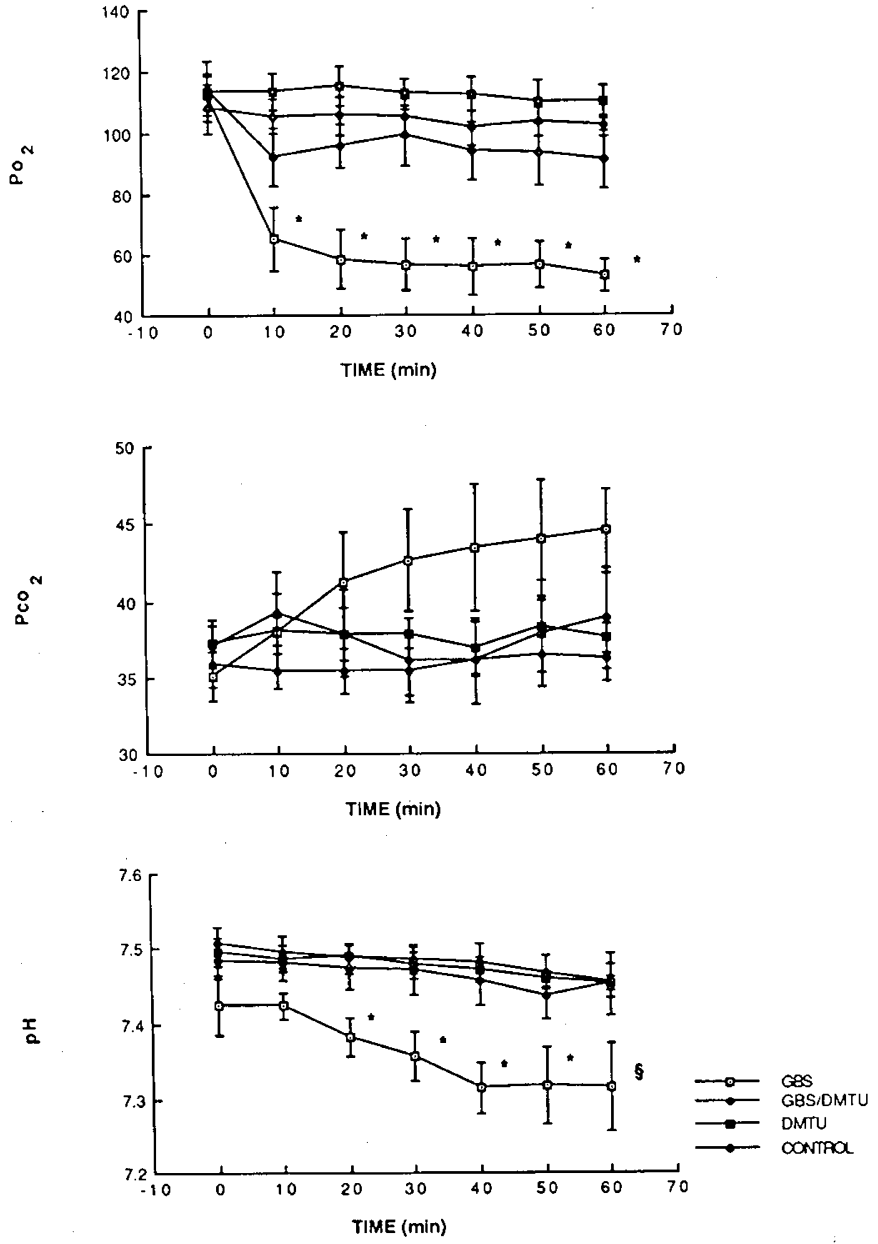

Fig. 3. Arterial $\mathrm{PO}_{2}, \mathrm{PCO}_{2}$, and $\mathrm{pH}$ in control piglets and piglets treated with GBS, DMTU, or GBS plus DMTU. $n=6$ for each group. Data are expressed as the mean $\pm \mathrm{SEM}$. ${ }^{*}$ Different from all other groups at $p<$ $0.05, \S$ different from both DMTU and GBS/DMTU at $p<0.05$.

A diverse array of oxidant-generating cell types may be involved in GBS-induced cardiopulmonary toxicity. Because polymorphonuclear leukocytes are found diffusely throughout the pulmonary vasculature in infants and experimental animals with GBS septicemia $(14,15)$, and because toxic oxygen metabolites derived from these cells have been implicated in a variety of models of acute lung injury (7), it is reasonable to suspect that polymorphonuclear leukocytes participate in the cardiopulmonary response to GBS septicemia. In direct support of this contention, Englehardt and co-workers (16) have shown that granulocyte depletion with hydroxyurea attenuates pulmonary mechanical and hemodynamic abnormalities evoked by GBS infusion in young lambs. Another cell type that may be involved in GBS-induced neonatal respiratory distress is the pulmonary intravascular macrophage, which, if functionally similar to alveolar macrophages (17), also elaborates toxic oxygen radicals. Although specific interactions between this cell type and GBS have not been reported, Warner et al. (18) have demonstrated in adult sheep that uptake of Pseudomonas aeruginosa by pulmonary intravascular macrophages is associated with evolution of respiratory distress. Interestingly, infusion of the same organism into rats, a species relatively devoid of pulmonary intravascular macrophages, fails to promote overt pulmonary dysfunction. Young piglets seem to resemble sheep: they exhibit both a brisk pulmonary response to GBS and a significant population of pulmonary intravascular macrophages, the density of which appears to increase during the first several weeks of extrauterine life (19). Finally, GBS also may interact directly with pulmonary

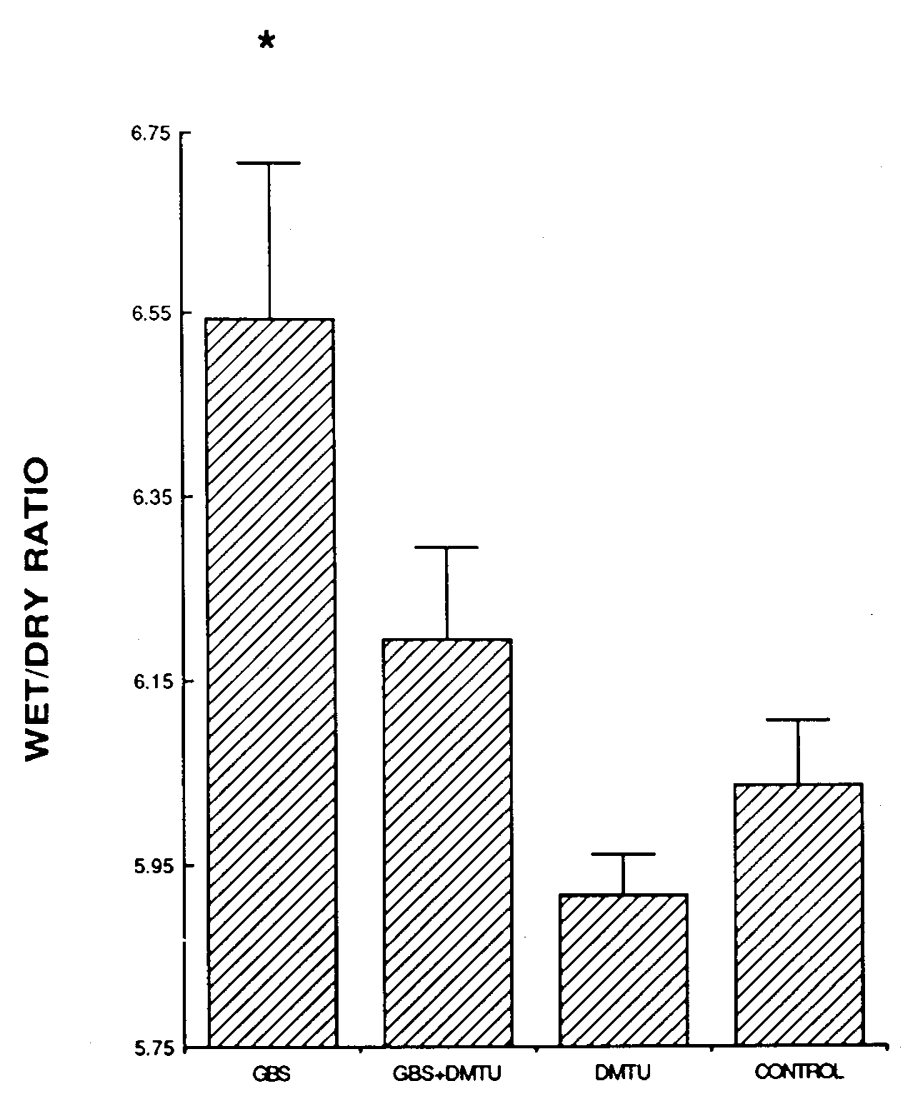

TREATMENT

Fig. 4. Postmortem lung wet-to-dry (wet/dry) weight ratios in control animals and in animals treated with GBS, DMTU, or GBS plus DMTU. $n=6$ for each group. ${ }^{*}$ Different from all other groups at $p<0.05$.

vascular endothelial cells to affect intracellular generation of oxidants with attendant endothelial dysfunction. This possibility, too, has not been examined in the specific case of GBS, but it is noteworthy that endotoxin and Salmonella minnesota promote oxidant generation in cultured endothelial cells $(11,12)$. Because DMTU is a relatively small, lipophilic molecule, it might be expected to scavenge intracellularly generated radicals while larger scavengers, such as superoxide dismutase or catalase, would be unable to do so. Perhaps a comparison of these three scavenger systems would prove valuable in localizing oxidant generation to extra- versus intracellular sites.

Previous reports indicate that arachidonic acid metabolites play central roles in the development of GBS-induced neonatal cardiopulmonary dysfunction. Compelling evidence supports involvement of cyclooxygenase products: GBS infusion is accompanied by accumulation of immunoreactive $\mathrm{TXB}_{2}$, and the acute pulmonary hypertensive response can be abrogated or reversed by cyclooxygenase inhibitors and/or thromboxane synthetase inhibitors (1-3). In support of a role for 5'-lipoxygenase products, Goldberg and coworkers (4-6) have demonstrated that FPL 57231, an inhibitor of peptidoleukotriene receptors, suppresses both airways and pulmonary hemodynamic abnormalities evoked by GBS. Results of the present study raise the issue as to how toxic oxygen radicals interact with arachidonic acid metabolites in GBS septicemia. One probable mechanism is that oxidants initiate production of eicosanoids which then serve as primary injurious mediators. Tate et al. (20) have shown that chemically generated oxygen radicals evoke thromboxane synthesis and thromboxane-dependent vasoconstriction in isolated, salt-solution-perfused rabbit lungs. Similarly, we (21) and others 
(22) have demonstrated in isolated salt-solution-perfused rat lungs that hydrogen peroxide provokes accumulation of $5^{\prime}$ lipoxygenase products and that blockade of either lipoxygenase or peptidoleukotriene receptors inhibits hydrogen peroxide-induced edema formation. Thus, in isolated lung preparations, toxic oxygen radicals appear to evoke eicosanoid-dependent changes which resemble those occurring in GBS-treated young piglets.

It is tempting to speculate that blockade of oxygen radicals and/or eicosanoids could serve as novel therapeutic approaches to GBS septicemia. One aspect of this possibility that warrants special consideration is whether blockade of either of these pathways disrupts host bacterial defense systems and thus exacerbates the bacteremia. This seems to be particularly relevant in the case of oxygen radical inhibition because oxidative killing is likely an important host-defense mechanism. Additional experiments will be necessary to resolve this issue.

In summary, results of the present study indicate that DMTU protects young piglets against GBS-induced pulmonary hypertension, pulmonary edema, and arterial hypoxemia and thereby implicate hydroxyl radicals in the cardiopulmonary consequences of GBS infection in this animal model. The cell type(s) responsible for oxidant production, the relation of oxidants to eicosanoid generation, and the specific role(s) of these mediators in GBS infection remain to be established.

\section{REFERENCES}

1. Runkle B, Goldberg RN, Streitfeld MM, Clark MR, Buron E, Setzer ES, Bancalari E 1984 Cardiovascular changes in Group B streptococcal sepsis in the piglet: response to indomethacin and relationship to prostacyclin and thromboxane A2. Pediatr Res 18:874-878

2. Truog WE, Sorenson GK, Standaert TA, Redding GJ 1986 Effects of a thromboxane synthetase inhibitor, dazmegrel (UK 38,485) on pulmonary gas exchange and hemodynamics in neonatal sepsis. Pediatr Res 20:491-486

3. Tarpey MN, Graybar GB, Lyrene RK, Godoy G, Oliver J, Gray BM, Philips JB 1987 Thromboxane synthetase inhibition reverses group B streptococcusinduced pulmonary hypertension. Crit Care Med 15:644-647

4. Goldberg RN, Suguihara C, Streitfeld MM, Bancalari A, Clark MR, Bancalari E 1986 Effects of leukotriene antagonist on the early hemodynamic manifestations of group B streptococcal sepsis in piglets. Pediatr Res 20:10041008

5. Suguihara C, Goldberg RN, Hehre D, Bancalari A, Bancalari E 1987 Effect of cyclooxygenase and lipooxygenase products on pulmonary function in group
B streptococcal sepsis. Pediatr Res 22:478-482

6. Goldberg RN, Suguihara C, Martinez O, Bancalari A, Clark M, Bancalari E 1988 The role of leukotrienes in the late hemodynamic manifestations of group B streptococcal sepsis in piglets. Prost Leuk Med (in press)

7. Brigham KL 1986 Role of free radicals in lung injury. Chest 89:859-863

8. Olson NC, Anderson DL, Grizzle, MK 1987 Dimethylthiourea attenuates endotoxin-induced respiratory failure in pigs. J Appl Physiol 63:2426-2432

9. Fox RB, Harada RN, Tate RM, Repine JE 1983 Prevention of thioureainduced pulmonary edema by hydroxyl radical scavengers. $J$ Appl Physiol 55:1456-1459

10. Fox RB 1984 Prevention of granulocyte-mediated oxidant lung injury in rats by a hydroxyl radical scavenger, dimethylthiourea. J Clin Invest 74:14561464

11. Brigham KL, Meyrick B, Berry LC, Repine JE 1987 Antioxidants protect cultured bovine endothelial cells from injury by endotoxin. J Appl Physiol 63:840-850

12. Ryan US 1987 Phagocytic properties of endothelial cells. In: Ryan US (ed) Endothelial Cells. CRC Press, Boca Raton, FL 21-43

13. Collins JC, Newman JH, Wickersham NE, Vaughn WK, Snapper JR, Harris TR, Brigham KL 1985 Relation of blood-free to blood-inclusive post mortem lung water measurements in sheep. J Appl Physiol 59:592-592.

14. Hemming VG, McCloskey DW, Hill HR 1976 Pneumonia in the neonate associated with group B streptococcal septicemia. Am J Dis Child 130:12311233

15. Rojas J, Larson LE, Brigham KL, Gray ME, Stahlman MT 1983 Pulmonary hemodynamic and ultrastructural changes associated with group B streptococcal toxemia in adult sheep and newborn lambs. Pediatr Res 17:10021008

16. Englehardt B, Sandberg K, Bratton D, Van Den Abbeele A, Grogaard J, Hellerqvist C, Sundell H 1987 The role of granulocytes in the pulmonary response to group B streptococcal toxin in young lambs. Pediatr Res 21:159165

17. Fell AOS, Cohn ZA 1986 The alveolar macrophage. J Appl Physiol 60:353369

18. Warner AE, Molina RM, Brain JD 1987 Uptake of bloodborne bacteria by pulmonary intravascular macrophages and consequent inflammatory response in sheep. Am Rev Respir Dis 136:683-690

19. Winkler GC, Cheville NF 1987 Postnatal colonization of porcine lung capillaries by intravascular macrophages: An ultrastructural morphometric analysis. Microvas Res 33:224-232

20. Tate RM, Vanbenthuysen KM, Shasby DM, McMurtry IF, Repine JE 1982 Oxygen radical-mediated permeability edema and vasoconstriction in isolated perfused rabbit lungs. Am Rev Respir Dis 126:802-806

21. Gillespie MN, Moore CG, Wright CE, O'Connor WN 1987 Salutary effects of prostaglandin $E_{1}$ in perfused rat lungs injured with hydrogen peroxide. $J$ Pharmacol Exp Ther 241:1-5

22. Burghuber OC, Strife RJ, Zirroli J, Henson PM, Henson JE, Mathias MM Reeves JT, Murphy RC, Voelkel NF 1985 Leukotriene inhibitors attenuate rat lung injury induced by hydrogen peroxide. Am Rev Respir Dis 131:778785 